\title{
LAS INTERFACES URBANO-FORESTALES: UN NUEVO TERRITORIO DE RIESGO EN ESPAÑA
}

\author{
Luis Galiana Martín \\ Departamento de Geografía. Universidad Autónoma de Madrid
}

\section{RESUMEN}

La expansión de la interfaz urbano-forestal en España, asociada a la creciente dispersión del sistema de asentamientos y a la progresión de la vegetación natural, constituye una dinámica territorial preocupante en relación con el problema de los incendios forestales. A pesar del incremento de episodios de incendios urbano-forestales, no existe una conciencia social ni una preocupación política sobre este asunto. Ello provoca un déficit normativo y de intervención sobre este nuevo territorio de riesgo.

Palabras clave: Dispersión urbana, incendios forestales, vulnerabilidad territorial, planificación espacial, gestión del territorio.

\section{ABSTRACT}

The wildland-urban interface: a new risk prone area in Spain. The spreading of the wildland-urban interface in Spain, related to the increasing sprawl of human settlements and also to the progression of natural vegetation, constitutes a worrying territorial dynamics in connection to the problem of wildland fires. Despite the increase in number of episodes of wildland-urban fires, there is neither social awareness nor political concern on this matter. This results in a shortage of laws and management on these new territories at risk.

Key words: Urban sprawl, wildfires, spatial vulnerability, spatial planning, land management.

Fecha de recepción: julio 2010.

Fecha de aceptación: febrero 2012. 


\section{LA DEFINICIÓN, DELIMITACIÓN Y CARACTERIZACIÓN DEL PROBLEMA}

Actualmente, uno de los elementos que más está contribuyendo a incrementar la vulnerabilidad territorial frente a los incendios forestales en España es el desarrollo de situaciones en las que estas manifestaciones de inestabilidad ambiental afectan o pueden afectar a edificaciones y zonas urbanizadas. Esta extensión de la amenaza a bienes y personas provoca, además de un incremento del riesgo, una creciente complejidad en las labores de prevención y, sobre todo, de extinción. Sin embargo, la percepción social sobre este nuevo territorio de riesgo sigue siendo débil. Ello explica que la consideración de esta realidad, a pesar de su importancia superficial creciente, no haya alcanzado la dimensión e intensidad necesarias (salvo contadas excepciones) para abordar un tratamiento específico desde las diferentes políticas públicas con capacidad para intervenir en estas cuestiones (forestal, urbanismo, ordenación del territorio, protección civil, etc.).

Los objetivos del presente artículo son dos. En primer lugar, realizar un breve estado de la cuestión, tanto del propio concepto de interfaz como sobre su tratamiento en la literatura científica. Y en segundo lugar, presentar la actualidad de esta realidad en España: distribución, episodios recientes de incendios, tratamiento normativo.

\section{La aparición y desarrollo del concepto de interfaz urbano-forestal}

Se entiende por interfaz urbano-forestal (IUF) la zona en la que el terreno forestal entra en contacto con zonas edificadas. Una definición simple para una realidad compleja, caracterizada por las múltiples interacciones e intercambios que se desarrollan entre dos subsistemas territoriales (el social/urbano y el natural/forestal) (Vince et al., 2005). A pesar de que el desarrollo de edificaciones en zonas de vegetación natural supone un reto ambiental en sentido amplio (fragmentación de hábitats, introducción de especies invasoras en espacios naturales, alteración del ciclo hidrológico, etc.) (Radeloff et al., 2005), este término se utiliza preferentemente desde la perspectiva de los incendios forestales. Con él se designa el escenario territorial de un nuevo fenómeno: el fuego urbano-forestal, un tipo de incendio que no sólo puede alcanzar, sino que además puede propagarse en el interior de los desarrollos edificatorios, lo que hace que surjan unos problemas específicos, sustancialmente distintos a los provocados por los incendios puramente forestales o urbanos (Pyne et al., 1996). Así pues, el territorio de interfaz es el susceptible de ser afectado por incendios forestales, pero al mismo tiempo constituye una fuente de peligro, pues se trata de un ámbito donde las probabilidades de ignición por causas humanas son mucho más frecuentes (Vilar et al., 2008).

En efecto, el interés por la IUF y su propia definición se relacionan directamente con su consideración como un territorio de riesgo frente a los incendios forestales. La primera constancia de la utilización del término se produce en California a mediados de los años 70 en relación con la especificidad de un tipo de incendio cada vez más frecuente (the urban/ wildland fire interface) (Butler, 1976). A pesar de la atención prestada a esta nueva emergencia por parte de los servicios antiincendios desde los años 60 , no será hasta mediados de los 80 cuando la concienciación alcance las esferas oficiales. Esto se produce en Estados Unidos tras un episodio de incendios catastróficos ocurridos en 1985 que afectaron muy especialmente a zonas habitadas (Davis, 1990). Se da comienzo a una nueva política de gestión del 
problema provocado por la creciente urbanización de ámbitos forestales (Walt, 1988), aceptando además que los fuegos de interfaz han dejado de ser un problema exclusivo de California (National Wildland/Urban Interface Fire Protection Iniative, 1987) (Foote et al., 1991).

De esta forma, desde hace ya más de veinte años comienza a generalizarse el uso de este concepto, que surge en Estados Unidos (primero en California, más tarde en otros estados) y que se extiende paulatinamente a otros ámbitos afectados por los mismos problemas (Australia, Canadá, Europa occidental). Un concepto directamente asociado al fenómeno de la urbanización dispersa y a la presión edificatoria proyectada sobre zonas forestales (Avalapati et al., 2005), y que se plantea esencialmente para definir una realidad dinámica (la frontera entre un proceso que avanza, la urbanización, sobre un territorio que es ocupado: los terrenos forestales). Y, como en todos los espacios de frontera, esta ocupación se salda asumiendo riesgos: en este caso, y de manera muy especial, la afección por incendios forestales.

\section{El tratamiento científico de la interfaz urbano-forestal}

En los últimos veinte años, la investigación sobre esta nueva realidad (en realidad, lo nuevo era la percepción de un territorio de riesgo sobre el cual había que actuar) ha tenido un fuerte desarrollo, multiplicándose las publicaciones sobre la misma. Y ya no sólo en EEUU, Australia o Canadá, sino también en Europa, donde varios proyectos de investigación sobre incendios forestales, y en ocasiones específicos sobre interfaces, han incluido este asunto entre sus prioridades ${ }^{1}$. El resultado de todo ello es que el de interfaz sea un concepto bien asentado, especialmente en la comunidad científica centrada en los incendios forestales, dando lugar incluso a la aparición de manuales que, de alguna manera, nos muestran hasta qué punto se han consolidado ciertos conceptos, prácticas y formas de tratamiento de esta realidad (Vince et al., 2005).

El tratamiento científico de las interfaces presenta un sesgo muy definido en relación con un objetivo común: su voluntad de contribuir a la resolución del problema territorial que supone la cada vez mayor importancia superficial de este fenómeno. Y en esta línea de puesta de manifiesto de la insostenibilidad de estos asentamientos se definen buen número de publicaciones que abordan los problemas para su delimitación y cartografía, su caracterización tipológica, el estudio de los efectos que provocan sobre los ecosistemas, las posibilidades de gestionar el riesgo en la interfaz, etc. (Avalapati et al., 2005).

\section{Los métodos de delimitación y caracterización}

Los primeros esfuerzos para identificar y cartografiar el fenómeno fueron llevados a cabo en EEUU en el marco de la planificación de incendios forestales, alentados por el incremento del riesgo provocado por su proliferación (al convertirse en la principal causa de pérdidas en los grandes incendios y de incremento de los gastos de extinción). Por este motivo, y

1 FIRE PARADOX (An innovative and integrated approach to wildland fire management), 2006-2010 (http://www.fireparadox.org/index.php); EUFIRELAB (Euro-mediterranean Wildland Fire Laboratory), 2003-2006 (http://www.eufirelab.org/index.php); WARM. (Wild land-urban Area Fire Risk Management), 2001-2004; FIRE STAR (Un sistema de ayuda a la decisión para la gestión del combustible y la reducción del riesgo de incendios en la interfaz urbano-forestal mediterránea), 2002-2003 (http://www.eufirestar.org/index.php); SPREAD (2003), ... 
con el objetivo de identificar las comunidades que vivían en zonas de interfaz, el Registro Federal definió oficialmente esta realidad, diferenciando dos tipos de situaciones (interfaz e intermix) ${ }^{2}$ y estableciendo una metodología de representación para las mismas (USDA \& USDI, 2001). Se trata de aproximaciones a pequeña escala, con la sección censal como unidad de análisis espacial, y sobre la que se representan los datos de densidad de viviendas procedentes del censo y de vegetación, definida a través de teledetección . De acuerdo a esta metodología, en el año 2000 se estimaba que el 9\% del territorio y el 39\% de las viviendas de EEUU se situaban en zonas de interfaz (Radeloff et al., 2005); y mediante la superposición de dos momentos (1990-2000) se detectaban las principales dinámicas de avance del fenómeno y los factores que las determinaban (Hammer et al., 2007; Zhang et al., 2008).

Junto a estos esfuerzos por determinar el alcance del fenómeno sobre amplios territorios, e igualmente vinculados a la gestión de riesgos, se han ido desarrollando métodos para delimitar con precisión y a gran escala estas situaciones que han permitido avanzar en la caracterización y diferenciación interna de las mismas. En este sentido, los trabajos acometidos desde el CEMAGREF de Aix-en-Provence constituyen el principal referente en la materia (Lampin-Maillet et al., 2010). Estos métodos permiten abordar de una manera más afinada la descripción y clasificación de las interfaces a partir del análisis de sus principales componentes: la intensidad de la ocupación humana (densidad y número de edificaciones) y las características de la vegetación (continuidad horizontal de la misma), y de sus posibles combinaciones (Lampin et al., 2006; Caballero et al., 2007). Aunque en general se vinculan al problema de los incendios forestales, estas clasificaciones pueden abordar de una manera más amplia el impacto de las interfaces sobre las zonas naturales (Dumas et al., 2008).

Junto a estas aproximaciones analíticas y estáticas (de aplicación general en cualquier ámbito espacial) tenemos otras más sintéticas y dinámicas (más apropiadas para abordar la variedad regional que adquiere el fenómeno), que vinculan los tipos de interfaz al carácter y evolución de los paisajes en los que se desarrollan (Galiana et al, 2011), con un objetivo común (establecer el nivel de riesgo asociado a cada uno de los territorios que acogen situaciones de interfaz). En esta misma línea de determinar los niveles de vulnerabilidad asociados a cada tipo de interfaz, se han elaborado tipologías de interfaces que tienen en cuenta especialmente la capacidad de propagación de los incendios por el interior de las zonas urbanizadas (Castellnou et al., 2005; Caballero et al., 2007).

\section{El tratamiento del riesgo en la interfaz urbanp-forestal}

El otro gran asunto sobre el que se enfoca buena parte de la literatura científica es la gestión del riesgo en la IUF. La necesidad de aplicar nuevas líneas de actuación, propias y diferenciadas para estos espacios, se plantea ante el convencimiento de la inevitabilidad del proceso y de su dinámica progresiva. Una acción positiva que pasa inevitablemente por dos grandes cuestiones: la concienciación de los residentes en estos espacios y una mayor consideración de esta situación de riesgo por parte de la planificación (tanto espacial como de protección civil) (Walt, 1988), y que se mueve por tanto entre la adopción de medidas de

2 La principal diferencia entre interfaz e intermix es, respectivamente, la posiblidad o no de establecer un límite claro entre el espacio edificado y el forestal. 
mitigación de forma voluntaria y las acciones de regulación obligatoria por parte de la administración, respectivamente.

A partir de finales de los años 80, algunas agencias oficiales (California Department of Forestry and Fire Protection) comienzan a realizar estudios basados en el análisis de estructuras afectadas por incendios para identificar medidas de defensa capaces de reducir la vulnerabilidad frente al fuego de las viviendas situadas en zonas de interfaz (Foote et al., 1991). Los factores clave identificados sobre los que se deberían adoptar medidas preventivas para disminuir la amenaza fueron la intensidad del fuego y el tipo de estructuras y su inflamabilidad, abriendo paso a intervenciones e investigaciones en estas líneas.

Son muy numerosos los trabajos dedicados a valorar la efectividad de los tratamientos del combustible en la IUF para reducir la intensidad del fuego en las inmediaciones de las estructuras edificadas (Reinhardt et al., 2008; Safford et al., 2009; Ager et al., 2010). La especificidad de dichas actuaciones, en relación con las especiales consideraciones paisajísticas aplicadas a estos ámbitos o la dificultad de emplear ciertas técnicas (fuego prescrito) por la afección a zonas densamente habitadas, son las principales conclusiones establecidas. No faltan valoraciones generales sobre estrategias integradas de mitigación de riesgos en estos ámbitos espaciales (Reams et al., 2005). Aunque en su mayor parte son aproximaciones efectuadas desde la realidad norteamericana, también pueden encontrarse referencias sobre las regiones mediterráneas (Proceedings, 2003).

La modelización de los comportamientos de los incendios, la valoración de la propagación en la proximidad de las estructuras edificadas y la ignición de las mismas conforma igualmente buena parte del trabajo y de los resultados de los proyectos de investigación previamente citados (WARM, EUFIRELAB, FIRE STAR, ...).

Son, sin embargo, menos numerosas las referencias que tratan de los problemas de los trabajos de extinción. Desde la presentación y análisis de experiencias (Castellnou et al., 2005), se abordan muy especialmente los asuntos referidos a los problemas causados por la población residente (evacuación, confinamiento, etc.) (Stephens et al., 2009; Haynes et al., 2010) y las necesidades de autoprotección (Beringer, 2000).

\section{DISTRIBUCIÓN DE LAS SITUACIONES DE INTERFAZ FORESTAL EN ESPAÑA Y EPISODIOS DE INCENDIOS}

A pesar de constituir una realidad que ha llegado a definir un verdadero paisaje de referencia para el monte mediterráneo en diferentes ámbitos del territorio español (ciertos tramos de la costa mediterránea: Costa Brava, Costa Blanca, Mallorca; las coronas metropolitanas más externas de Madrid, Barcelona o Valencia), se conoce poco sobre la magnitud superficial del fenómeno y su distribución espacial. Lo que parece innegable es que su presencia creciente en el territorio se está traduciendo en un incremento de los episodios de fuego urbano-forestal en España, como por ejemplo los que acompañaron a las grandes oleadas de incendios que afectaron a Canarias (2007) o Galicia (2006), y que son cada vez más comunes en ámbitos fuertemente urbanizados como Madrid y Cataluña.

Este incremento de la vulnerabilidad viene determinado por una evolución territorial en la que están presentes dos factores. Por un lado, una dinámica de progresión de la vegetación que se traduce en una mayor continuidad (tanto vertical como horizontal) de las formaciones 
forestales, lo que facilita la propagación de los incendios (Badia et al., 2002). Y, por otro, por una marcada tendencia a la dispersión de los procesos de urbanización, especialmente en ámbitos metropolitanos y litorales. De la interacción entre ambos factores se deriva una multiplicación de las situaciones de interfaz urbano-forestal.

\section{La evolución de los territorios de interfaz}

El primer intento de cartografía de esta realidad en España a escala nacional ha sido realizado mediante la delimitación de las zonas de contacto entre las superficies forestales y las zonas urbanas en los años 1987 y 2000 (Montiel y Herrero, 2010) ${ }^{3}$. Para ello se han utilizados los datos sobre cubiertas del suelo proporcionados por el proyecto Corine Land Cover, junto con un mapa de poblamiento elaborado por la empresa TRAGSATEC (Galiana et al., 2009). Los datos manejados en este trabajo nos hablan de aproximadamente 1,1 millones de ha de zonas de interfaz urbano-forestal en 2000, con un incremento del 6,8 por 100 desde 1987 (Ver Figura 1).

El mapa elaborado nos permite apreciar como estas situaciones de contacto alcanzan especial densidad en Galicia, Asturias o Canarias, ámbitos caracterizados por la elevada dispersión de su sistema de asentamientos rurales. Afecta especialmente también a los ámbitos con mayores niveles de urbanización. Así ocurre en las coronas metropolitanas de Madrid, Barcelona y, en menor medida, de Valencia; igualmente notables son las concentraciones que aparecen en el litoral mediterráneo con mayor intensidad del uso turístico (comarcas de la Marina en Alicante, del Ampurdán en Girona o de la Costa del Sol occidental en Málaga).

Figura 1

VEGETACIÓN FORESTAL Y ZONAS DE INTERFAZ URBANO-FORESTAL (2000)

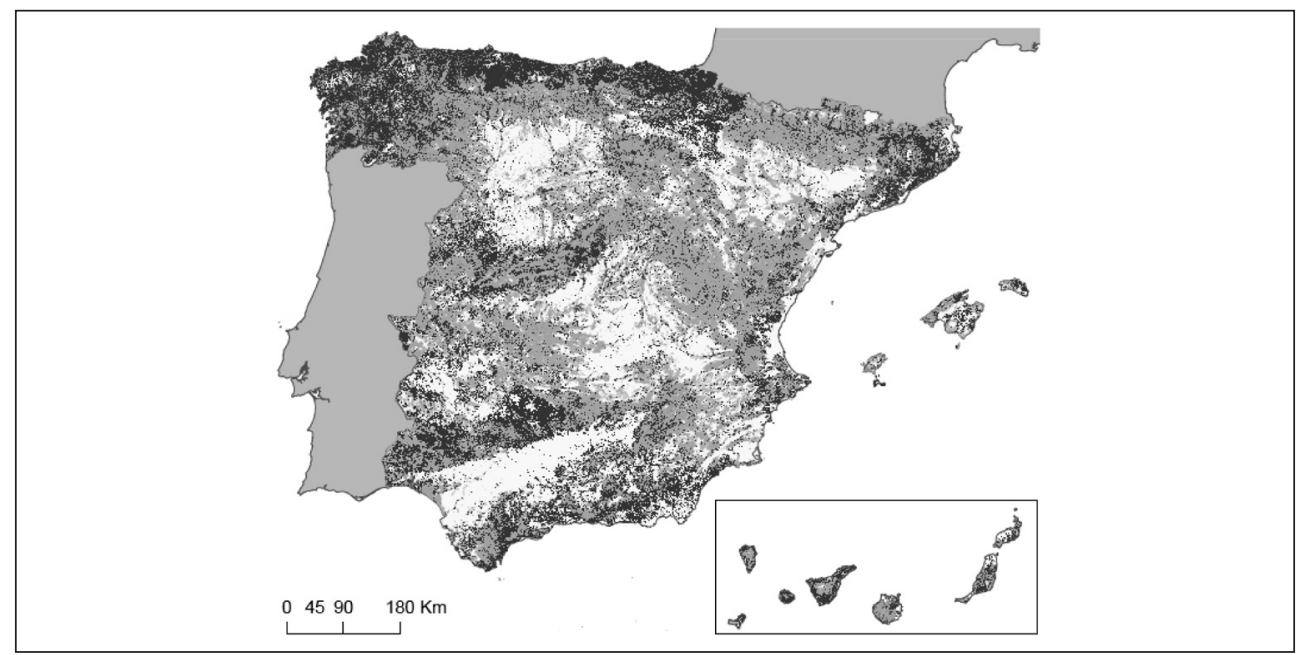

Fuente: Montiel y Herrero, 2010.

3 Una representación cartográfica de las zonas de IUF ha sido realizada para la región metropolitana de Barcelona (Badia et al., 2010). 


\section{La creciente urbanización del territorio, principal razón del avance de las interfaces}

La proliferación de las situaciones de interfaz se relaciona principalmente con los procesos de urbanización que afectan a un determinado territorio y, en concreto, con los patrones espaciales que adoptan dichos procesos. El intenso proceso de suburbanización propio de la ciudad americana, que estaba en el origen de la aparición del fenómeno de las interfaces (primero en California y más tarde en otros estados), llega a Europa (y a España) con varios decenios de retraso. Pero, a pesar de su aparición más tardía, se trata de un proceso bien consolidado, que se ha extendido por las zonas de mayor incremento de la urbanización de Europa occidental durante los últimos cincuenta años (European Commission, 2006; Font, 2007). La distribución de la IUF y su importancia superficial en 2000 en España (Fig. 1) muestra claramente esta tendencia en los entornos metropolitanos de Madrid y Barcelona, pero también aparece claramente relacionada con el fenómeno de la segunda residencia en los ámbitos de fuerte urbanización turística del litoral mediterráneo.

La dinámica experimentada entre 1987 y 2000 (y que se continúa en los años siguientes de acuerdo a los avances de datos aportados por el proyecto Corine para 2006) no hace sino reforzar y extender el proceso. La intensa transformación del territorio experimentada, con un incremento de las superficies artificiales de 240.000 ha (lo que supone un 29,5\% de incremento, frente al 5,4\% experimentado por los demás países europeos representados en el proyecto Corine) (Observatorio, 2006), ha tenido como principal motor el fuerte crecimiento del parque inmobiliario. En buena medida, este crecimiento de los espacios residenciales ha intensificado el proceso de dispersión urbana, en tanto que la mayor parte de la producción de viviendas se ha realizado fuera de la ciudad y con tipologías de baja densidad (Brandis, 2007). Fuerte crecimiento de las zonas urbanizadas que se puede generalizar para todo el territorio nacional, pero que ha sido especialmente intenso en determinadas zonas (ámbitos metropolitanos y, en general, aglomeraciones urbanas y zonas litorales) y con determinados tejidos residenciales (urbanizaciones exentas y/o ajardinadas) ${ }^{4}$. El fenómeno de la dispersión ha dejado de ser un proceso metropolitano (afectando también a ciudades medias y pequeñas), se ha ampliado a ámbitos litorales hasta ahora poco urbanizados y llega a afectar incluso a ámbitos claramente rurales de montaña (Mata, 2007).

\section{La progresión de la vegetación natural}

El otro elemento que puede contribuir a la expansión de las interfaces, junto al avance de la urbanización, es la progresión de la vegetación natural en detrimento de las superficies cultivadas. Sin embargo, la evolución de los usos del suelo entre 1987 y 2000 a partir de los datos del proyecto CORINE refleja una pérdida neta de superficie forestal de 250.783 ha. Una pérdida escasa del uso forestal en términos porcentuales (1 por 100), que recoge importantes transformaciones internas: pérdida de masas boscosas, sobre todo de coníferas, en buena medida por tala de las mismas o por incendios (pasan a formaciones arbustivas); reforestaciones, progresión de la vegetación natural, etc. Las principales pérdidas netas se

4 En la Comunidad de Madrid, la superficie ocupada por urbanizaciones exentas o ajardinadas se ha incrementado un 36,5 por 100 entre 1987 y 2000, y un 48,6 por 100 en la Comunidad Valenciana (Observatorio, 2006). 
centran en las formaciones de matorrales esclerófilos y pastizales, y son debidas a roturaciones agrícolas, con especial incidencia en las sierras del sur peninsular, y, en menor medida, a procesos de urbanización (Observatorio, 2006).

No obstante, este descenso de la superficie forestal no se deduce de otras aproximaciones estadísticas. Así, de las cifras de los sucesivos inventarios forestales nacionales (IFN), y con toda la prudencia que las diferentes metodologías de representación cartográfica pueden provocar en la comparación de los datos finales, puede hablarse más bien de estabilización cuando no de ligero aumento de la superficie forestal en España, que pasaría de 25.984.063 ha en 1995-96 (51,4 por 100 de la superficie total) (II IFN) a 27.527.974 ha en 2006-07 (54,4 por 100) (III IFN) ${ }^{5}$. Lo que parece que tiene menos discusión es el avance en otro elemento de la progresión de la vegetación natural que al principio señalábamos: la densificación forestal, que hace referencia tanto al aumento del número de pies arbóreos por ha, como a otros elementos de la biomasa forestal (cobertura del suelo por matorral bajo arbolado, espesor y cobertura de la hojarasca, etc.). Cambios cualitativos de especial significación, que también afectan al aumento de la continuidad de los usos forestales en ámbitos especialmente sensibles, como son los ruedos agrícolas de las poblaciones o las zonas periurbanas y litorales, tanto en la costa mediterránea como atlántica.

De esta forma, la frontera de la interfaz urbano-forestal se desplaza no sólo en función de procesos de urbanización irresponsables sobre ámbitos de alto riesgo potencial, sino también de manera más sigilosa, como resultado de la progresiva pérdida del uso agrícola en ámbitos especialmente sensibles, que provocan alteraciones sustanciales en las relaciones entre asentamientos humanos (que nunca antes habían tenido una percepción de estar situados en zonas de riesgo de incendios forestales) y su entorno inmediato.

\section{El problema en España: los episodios recientes de fuego urbano-forestal}

El cambio en las características de los incendios forestales en España y su vinculación a determinados factores de largo alcance (cambio climático, situación estructural de los montes) o de naturaleza más coyuntural (nuevas prioridades en la política forestal, mayor desarrollo de la capacidad de lucha) constituye un lugar común del debate más reciente sobre este problema ambiental (Greenpeace, 2009). Resulta difícil aislar cualquiera de estos elementos y establecer su grado de influencia en la definición de los nuevos escenarios sobre los que se desarrolla el fuego forestal en España. Parece, en cualquier caso, que el consenso avanza sobre determinadas cuestiones: se está experimentando un aumento del número de periodos críticos de fuego como consecuencia del cambio climático; la capacidad de propagación de los incendios por la cubierta forestal, en relación con la mayor continuidad y densidad de la misma, es cada vez mayor; los innegables avances en los sistemas de extinción se traducen en una disminución del número e importancia de los incendios de baja y mediana intensidad. Como consecuencia de todo ello resulta una importancia relativa cada vez mayor de los grandes incendios forestales que, desarrollados en condiciones meteorológicas extremas, quedan fuera de la capacidad de extinción; presentan el componente añadido de la alta intensidad,

5 http://www.mma.es/portal/secciones/biodiversidad/inventarios/ifn/index.htm 
convirtiéndose en el principal problema ambiental de ciertos territorios (Cataluña, Canarias) (González y Pukkala, 2007).

Uno de los elementos que acompañan esta evolución general de los incendios en los últimos decenios es la aparición y progresiva importancia del fuego urbano forestal, aspecto ligado de manera significativa a los últimos episodios catastróficos acaecidos en España (Cataluña, 2003; Galicia, 2006; Canarias, 2007). En estos eventos, la numerosa población afectada hizo necesarias evacuaciones masivas que provocaron una gran complejidad de las actuaciones de extinción, que dejaron de ser meros incendios forestales para acabar convirtiéndose en verdaderas emergencias de protección civil.

Los trabajos de análisis de incendios acometidos desde el Grup de Recolzament d'Incendis Forestals (GRAF) de la Generalitat de Cataluña han conducido a una modelización del comportamiento de los grandes incendios forestales durante los últimos 40-50 años, cuyas conclusiones pueden considerarse de carácter general. Según este modelo evolutivo, que pone en relación las características de los incendios con los cambios de la disponibilidad del combustible en el monte (continuidad, densidad) y con las transformaciones territoriales experimentadas (abandono agrícola, pérdida de funcionalidad del monte, urbanización dispersa, etc.), es posible definir cuatro generaciones de incendios (Rifà y Castellnou, 2007). De acuerdo a este modelo, en Cataluña (y en general en la Europa mediterránea) la tercera generación de incendios que aparece en los años 90 (fuegos de copas que superan la capacidad de intervención táctica; aparición de múltiples focos secundarios con avance a saltos a largas distancias) manifiesta un comportamiento preocupante en la década siguiente: la posibilidad de propagar sobre otro tipo de combustibles (urbanizaciones), cada vez más numerosas en relación con los procesos de urbanización dispersa. Ello permite hablar de una nueva generación de incendios, la cuarta, con nuevas dificultades para los servicios de extinción (Castellnou et al., 2005).

En efecto, algunos de los incendios acaecidos en Cataluña en el singularmente cálido agosto de 2003 presentaron estas características (Sant Llorenç Savall, 4.579 ha; Maçanet, 1.279 ha; Platja d'Aro, 429 ha). Las condiciones meteorológicas extremas de humedad y temperatura, combinadas con viento y fuerte inestabilidad, dieron lugar a fuegos de copas con múltiples focos secundarios que propagaron hacia las urbanizaciones y las atravesaron (Castellnou et al., 2005).

Diferentes características presentó el episodio de incendios forestales vivido por Galicia entre el 4 y el 13 de agosto de 2006 (alrededor de 80.000 ha afectadas, 4 fallecidos). Absolutamente sin precedentes, se caracterizó por su rápida y extendida conversión en un problema de seguridad ciudadana y de orden público al tratarse, en buena medida, de fuegos urbanoforestales que provocaron una gran alarma social y un sentimiento de inseguridad (Informe, 2006). Al gran número de incendios (2.055) y su simultaneidad (picos de 300 incendios activos, con una media de 5 focos), se añadía el componente del riesgo a la población residente: el 51 por 100 de los incendios fueron declarados de nivel 1 y 2 (aquellos con riesgos para vidas humanas y bienes de naturaleza no forestal) (Xunta de Galicia, 2006). Un total de 9.262 entidades de población fueron afectadas, localizadas principalmente en las zonas costeras de las provincias de A Coruña (Costa da Morte, comarca de Compostela) y Pontevedra (Salnés, comarca de Vigo), con un perímetro de interfaz urbano-forestal de 788.160 metros lineales (Xunta de Galicia, 2006) (Figura 2). 
Figura 2

LOCALIZACIÓN DE LOS INCENDIOS FORESTALES ENTRE EL 4 Y EL 15 DE AGOSTO DE 2006

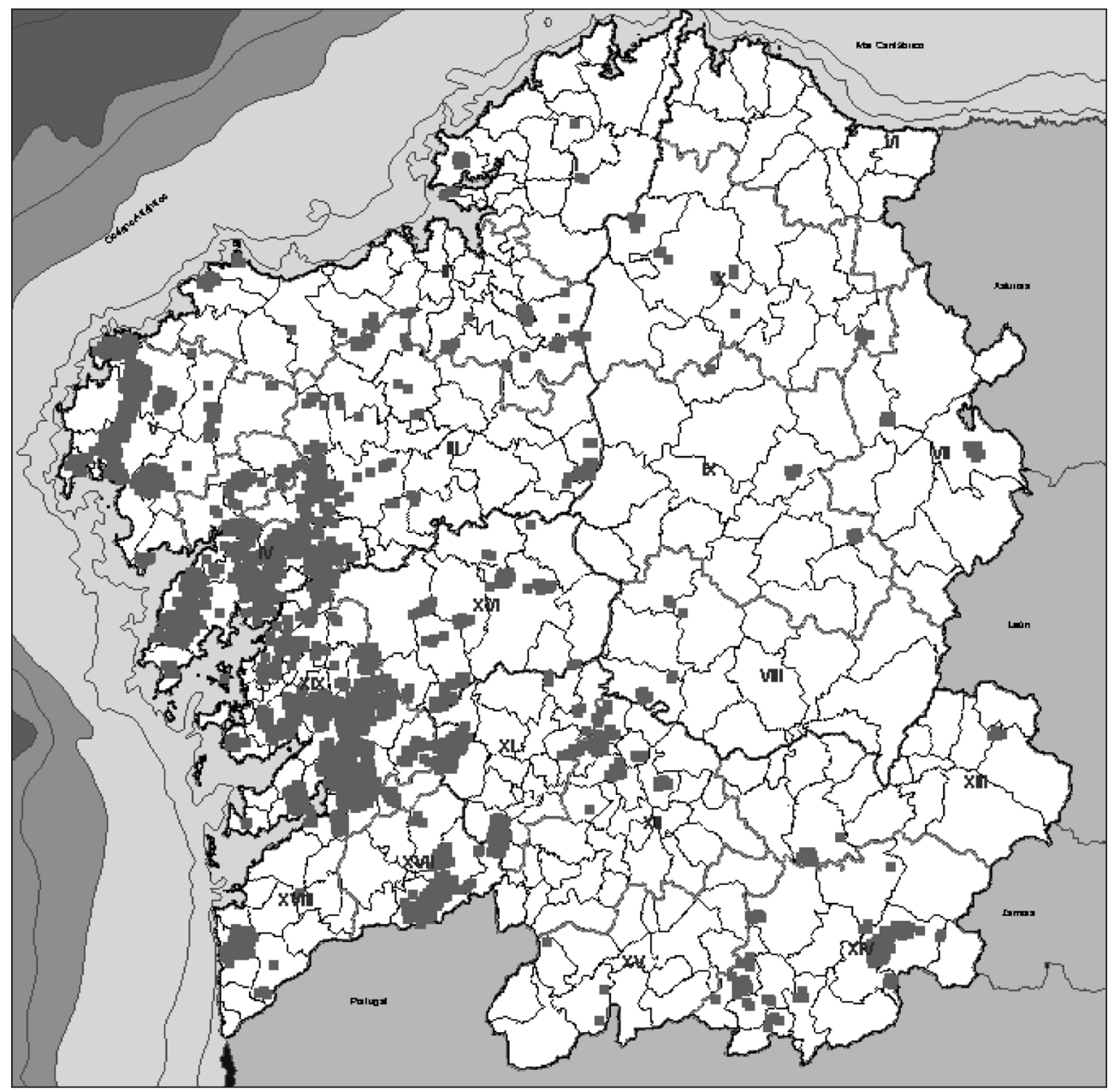

Fuente: Xunta de Galicia, 2006.

Dos grandes incendios originados en Tenerife (Los Realejos, 18.095 ha) y Gran Canaria (Tejeda, 19.190 ha) a finales de julio de 2007 calcinaron una superficie mayor que la registrada en el conjunto del archipiélago por todos los incendios forestales ocurridos en los veintidós años anteriores (MMARM, 2008). Junto a la importancia relativa extraordinaria que alcanzan un reducido número de sucesos que quedan fuera de la capacidad de extinción, este episodio puso de manifiesto igualmente el componente de emergencia de protección civil que los incendios forestales pueden llegar a causar, al afectar a un gran número de entidades de población y provocar el desalojo de más de 12.000 personas. Iniciados en grandes masas de pino canario, propagaron igualmente por los entornos agrícolas de los caseríos que, 
privados del mantenimiento que tradicionalmente se realizaba en torno a las zonas habitadas, fueron pasto de las llamas, que alcanzaron y atravesaron varios núcleos rurales.

Sin alcanzar el dramatismo de estos periodos, los ejemplos de grandes incendios que se convierten en emergencias de protección civil al afectar a zonas urbanizadas se han ido sucediendo en los últimos veranos (Turre, Almería, julio de 2009). Es el reflejo de una mayor vulnerabilidad territorial que se asocia a la creciente importancia que la urbanización residencial dispersa adquiere en el territorio español, pero que también tiene que ver con una progresión de la vegetación natural y una menor gestión de los entornos de muchos núcleos rurales. Todo ello supone un avance del territorio de riesgo sobre el que es necesario actuar desde diferentes perspectivas.

\section{EL TRATAMIENTO DE LA INTERFAZ URBANO FORESTAL}

La necesidad de gestionar la IUF se plantea desde varias perspectivas, que abarcan tanto las fases de prevención como de extinción de las emergencias, y que tienen que ver con la actuación abordada desde diferentes intervenciones públicas (planificación espacial, política forestal y de incendios, protección civil). Desde el punto de vista de la prevención, el tratamiento debería enfocarse, en primer lugar, a evitar su proliferación; sin embargo, los escasos esfuerzos realizados en este sentido se encaminan de manera prioritaria al tratamiento de la realidad existente. Queda también por abordar, de manera específica para estos territorios, la propia planificación de la emergencia, una labor en la que tan importante es la concienciación y autoprotección de los residentes en la interfaz como el trabajo de los profesionales implicados.

\section{La responsabilidad de la planificación espacial en el incremento de la vulnerabilidad territo- rial}

La planificación espacial en sentido amplio (ordenación del territorio, urbanismo) es, en última instancia, la responsable de la proliferación de interfaces. La vulnerabilidad de las áreas pobladas frente a los desastres naturales es el efecto de una mala planificación territorial (Fleischhauer et al., 2007), que no ha incorporado una pautas de uso sensato del territorio. Sin embargo, sobre esta realidad ya existente poco se puede realizar desde la planificación espacial. La principal aportación en este sentido sería frenar su progresión, adoptando un modelo territorial más prudente capaz de incorporar las relaciones entre las decisiones sobre usos del suelo y los regímenes del fuego presentes en un determinado territorio (Pincetl et $a l ., 2008)$. Para ello, sin embargo, deben superarse ciertos problemas de partida.

\section{Escasa consideración del riesgo como elemento asociado a la urbanización dispersa}

Los procesos de urbanización de baja densidad, y la percepción que se tiene de ellos como uno de los principales componentes de la insostenibilidad territorial que caracteriza los procesos de desarrollo más recientes, no incorpora entre los atributos negativos habitualmente enumerados (ineficientes económica y funcionalmente, socialmente segregadores, ambientalmente nocivos) (Indovina, 2007, entre otros) su contribución al incremento de la vulnerabilidad de amplios territorios frente al riesgo de incendios forestales. 
Y ello no ocurre de manera general, ni tampoco en zonas fuertemente castigadas por los incendios urbano forestales (como por ejemplo, Galicia): no se cumple la máxima del proceso basado en desastres, según la cual la intensidad de la atención prestada a los peligros naturales depende de las experiencias recientes, minusvalorando la aproximación histórica de largo alcance propia de las evaluaciones científicas del peligro. Así, por ejemplo, frente al episodio de incendios en Galicia de agosto de 2006, la ley territorial gallega de $2007^{6}$ aborda el problema de la dispersión en estos términos:

«En las últimas décadas el modelo tradicional de asentamiento de la población en Galicia está siendo sustituido por otro modo de ocupación indiscriminada del territorio que tiende a un constante crecimiento urbano renunciando a la regeneración de los tejidos urbanos consolidados.

Este modo de ocupación del territorio (...) causa graves perjuicios económicos, sociales y ambientales, como son, entre otros, la ineficiencia económica por los elevados costes energéticos, de construcción y mantenimiento de las infraestructuras y de prestación de los servicios públicos; la segregación social y espacial; la contaminación atmosférica y de las aguas; la pérdida de espacios de cultivo y de la biodiversidad; la degradación del paisaje y del patrimonio cultural, y la pérdida de la identidad territorial.»

Aspectos negativos todos ellos importantes, pero a los que no se incorpora el incremento del riesgo de incendios forestales (por aumento de la vulnerabilidad poblacional) provocado por este modelo de asentamientos. Y todo ello a pesar de que desde la política de prevención de incendio sí se insistía en este problema específico, como veremos más adelante.

\section{La necesaria aproximación sectorial al problema}

De acuerdo a la normativa general urbanística, la planificación espacial debe incorporar en sus determinaciones la delimitación de los territorios de riesgo a través del informe de sostenibilidad ambiental de los instrumentos de ordenación ${ }^{7}$, sobre los que se establece una regulación genérica de restricción de los usos edificatorios (clasificación como Suelo rural). Sin embargo, de acuerdo a la anterior normativa de 1998, la definición de los territorios de riesgo debe estar acreditada por la planificación sectorial ${ }^{8}$, que es la responsable de su evaluación, de manera que sólo sus mapas poseen una base legal vinculante? .

Ahora bien, ¿se adecuan estos mapas a la finalidad (establecer zonas no urbanizables) y a la escala territorial de análisis (regional o local)? Evidentemente, se trata de mapas elaborados con otras finalidades (prevención y extinción de incendios), que no cumplen los requerimientos de la planificación espacial. Se deberían, por lo tanto, fijar unos estándares mínimos

6 Ley 6/2007, de 11 de mayo, de medidas urgentes en materia de ordenación del territorio y del litoral de Galicia.

7 Ley 8/2007, de 28 de mayo, de suelo, arts. 12.3.a) y 15.2.

8 Ley 6/1998, de 13 de abril, sobre régimen del suelo y valoraciones.

9 Entre otras: Ley 7/2002, de 17 de diciembre, de Ordenación Urbanística de Andalucía (Art. 46.2 .a); Decreto Legislativo 1/2004, de 22 de abril, por el que se aprueba el texto refundido de las disposiciones legales vigentes en materia de ordenación del territorio y urbanismo del Principado de Asturias (Art. 58.2); Ley 2/2001, de 25 de junio, de Ordenación Territorial y Régimen Urbanístico del Suelo de Cantabria (Art. 108.1.a); Ley 9/2001, de 17 de julio, del Suelo de la Comunidad de Madrid (Art. 16.1.a). 
para el trazado de mapas de peligro que permitieran determinar que algunas actividades de asentamiento futuras están prohibidas (Fleischhauer et al., 2007). Y ello a diferentes escalas:

- Regional. Marco general para otros planes y programas de mayor detalle; basta con identificar las áreas potencialmente amenazadas para evitar, por ejemplo, más actividades de asentamiento.

- Local. Se necesita una evaluación de peligros más detallada. Se debe concebir como una base vinculante para las licencias de construcción individuales.

Ello permitiría valorar el alcance territorial del peligro con los usos del suelo que se superponen. A partir de esta base, se podrían elegir estrategias de gestión de riesgos específicas para áreas propensas a ciertos peligros: conservación de área libres de desarrollos, restricción de ciertos usos especialmente sensibles, establecimiento de condiciones de edificación más restrictivas (alturas, materiales), etc. (Fleischhauer et al., 2007).

\section{Las dificultades técnicas para su tratamiento y la necesidad de nuevos enfoques}

Junto a los problemas de percepción y dependencia de la planificación sectorial, la planificación espacial se enfrenta a evidentes limitaciones para hacer frente a esta nueva situación territorial desde respuestas técnicas codificadas. Como en otros asuntos, no parece que puedan plantearse soluciones estandarizadas para procesos poco conocidos, insuficientemente formalizados (Font, 2003).

Las posibilidades de gestión del territorio del riesgo por parte de la planificación espacial (restricción de los procesos de asentamiento, regulación de usos) pasan necesariamente por un ejercicio de zonificación. Y ello a pesar de las limitaciones y críticas a este tipo de prácticas, por lo que supone de anticipación normativa a largo plazo del modelo territorial; críticas que gravitan esencialmente sobre su concepción estática, frente a una realidad territorial que es esencialmente dinámica, y sobre su incapacidad para anticipar la evolución a medio plazo de los fenómenos de transformación del territorio (Ezquiaga, 2009)

Evidentemente, la gestión territorial a partir de la definición de categorías de ordenación no puede reposar sobre la idea de una imagen final e inalterable del territorio, sino que por el contrario debe apoyarse en la consideración de las dinámicas territoriales (urbanización dispersa, avance de la vegetación natural) claves para la definición de los territorios de riesgo. En este sentido, la planificación debe avanzar también en la adopción de una perspectiva estratégica, orientada a la detección y regulación de los procesos con incidencia espacial más relevantes e innovadores. De esta forma el plan debe jugar su papel de referente racionalizador de los procesos territoriales, territorializando y coordinando en el espacio y en el tiempo la gestión frente a los riesgos.

\section{La planificación sectorial (política forestal y de incendios forestales): una incorporación reciente e insuficiente del concepto a la normativa y a la práctica}

La regulación de las medidas de prevención frente a los incendios forestales en España se formulan de manera general, y teniendo siempre en cuenta las especificidades regionales propias de las diferentes Comunidades Autónomas (CCAA), desde normativas específicas, y sólo de manera subsidiaria desde la legislación forestal. A partir de mediados de los años 
90, las CCAA comienzan a elaborar planes de defensa contra incendios que aglutinan la dispersión normativa anterior, al tiempo que incorporan nuevas disposiciones, especialmente la Directriz Básica de Planificación de Protección Civil de Emergencia por Incendios Forestales (1993).

Es precisamente en el marco de esta norma cuando se empieza a definir un tratamiento específico del problema de las interfaces en España. En concreto, al señalar la obligación de redactar Planes de autoprotección «para núcleos de población aislada, urbanizaciones, campings, etc., que se encuentren ubicados en zonas de riesgo» ${ }^{10}$. Sin embargo, esta obligación genérica no ha tenido apenas relevancia en la mayor parte de CCAA, que no han desarrollado de manera general este tipo de planes $^{11}$.

Por su parte, la legislación forestal ha incorporado finalmente el concepto de IUF, si bien de manera amplia e imprecisa, vinculándolo al problema de los incendios. La ley de Montes estatal de 2003 se refiere a estos terrenos como aquellos que incluyen «las urbanizaciones, otras edificaciones, obras, instalaciones eléctricas e infraestructuras de transporte que están situadas en terrenos forestales y sus inmediaciones, que puedan implicar peligro de incendios o ser afectadas por estos»; y queda en manos de las Comunidades Autónomas identificar con mayor precisión estos espacios, establecer las normas de seguridad necesarias, así como la regulación de actividades que puedan generar riesgo de incendio ${ }^{12}$.

A partir de este marco general, algunas CCAA han profundizado en el tratamiento específico del problema asociado a este territorio. Los avances en la materia se han desplegado básicamente en dos direcciones: por un lado, y de manera generalizada, en la planificación y gestión específica de este espacio; por otro, y con carácter más incipiente pero con una previsible mayor importancia de cara a la prevención, en la valoración de la vulnerabilidad territorial inducida por la urbanización en terreno forestal, con las implicaciones que ello debe conllevar en otras actuaciones públicas (ordenación urbanística y protección civil, especialmente).

\section{La gestión del espacio de interfaz}

Los avances concretos en esta materia se han producido de manera muy selectiva, en relación con la prioridad otorgada a este asunto por parte de algunas CCAA. En este sentido, Cataluña ha desempeñado un papel pionero, siendo la primera en establecer actuaciones específicas de prevención en la IUF. La importancia que la urbanización en el medio forestal alcanza en esta comunidad (1.100 urbanizaciones, con unas 45.000 ha de interfaz) y la creciente importancia del fuego urbano-forestal están detrás del interés mostrado por la administración pública catalana en desarrollar un sistema de gestión eficaz de la interfaz urbano-forestal (soporte normativo y subvenciones) (Terés et al. 2007).

10 Orden de 2 de abril de 1993 por la que se publica el Acuerdo del Consejo de Ministros que aprueba la Directriz Básica de Planificación de Protección Civil de Emergencia por Incendios Forestales (3.5.1).

11 En la Comunidad de Madrid, una de las más afectadas por este problema, hasta 2009 sólo se había redactado el Plan de autoprotección de la Urbanización Las Cuestas (Galapagar) (Servicio de Bomberos de la Comunidad de Madrid).

12 Ley 43/2003, de 21 de noviembre, de Montes (Art. 44.3). 
Ya en 1995 se publicaba un decreto de medidas específicas que afectaba a «las urbanizaciones sin continuidad inmediata con la trama urbana (...) situadas a menos de 500 metros de terrenos forestales, así como a las edificaciones e instalaciones aisladas en terreno forestal, excluyendo a las edificaciones e instalaciones destinadas a explotaciones agrícolas y ganaderas», que tuvo grandes problemas jurídicos y prácticos de aplicabilidad (elevado coste de las actuaciones y necesidad de actuar sobre fincas forestales) ${ }^{13}$. Tras este antecedente, en el año 2003 se publica una nueva norma dirigida al mismo ámbito, que establece la obligación de tener una franja de protección perimetral de $25 \mathrm{~m}$ con una carga de combustible reducida, así como a mantener limpios de vegetación los solares libres y viales de acceso, disponer de una red de hidrantes, además de poseer el correspondiente Plan de autoprotección ${ }^{14}$. En 2005 se publica el correspondiente Decreto para el desarrollo de dicha ley, proponiendo un régimen de subvenciones por parte de la Generalitat para los trabajos de apertura y mantenimiento de las franjas perimetrales, que están siendo ejecutados principalmente por los Ayuntamientos directamente concernidos (Terés et al., 2007). No obstante, su ejecución no ha dejado de plantear problemas, derivados tanto de la interpretación del concepto de urbanización como de la falta de recursos técnicos y económicos (Badia et al., 2010).

La obligación de ejecutar fajas de seguridad de anchura variable para reducir la intensidad del incendio, junto con otras acciones complementarias, es el planteamiento más común para la gestión de este espacio. Andalucía, Aragón, Canarias, Castilla-La Mancha, La Rioja, Madrid o la Comunidad Valenciana han establecido esta obligación, con anchuras variables (15 a $25 \mathrm{~m}$ ). El ejemplo de Extremadura es tal vez el que alcanza una mayor complejidad técnica. Los denominados Planes Periurbanos de Prevención de incendios forestales, integrados en el Plan PREIFEX ${ }^{15}$, plantean la ejecución de una franja periurbana perimetral (400-200 m a partir del límite del suelo urbano, en función de su ubicación o no en Zona de Alto Riesgo) cuya responsabilidad recae en los ayuntamientos. Entre otras actuaciones (caminos, puntos de agua), en dichas franjas se realizarán dos áreas cortafuegos de $80 \mathrm{~m}$ : una circundando al suelo urbano (para evitar propagaciones hacia el monte), y otra exterior, para evitar que el incendio forestal amenace las poblaciones; en ellas se reducirá el combustible, eliminando siempre el pasto (García y Carrascal, 2007).

En el caso de Galicia, la importancia creciente del problema se traduce en un tratamiento normativo más amplio y específico, atento a las especiales consideraciones de la dispersión propia del sistema de asentamientos rurales y a las dinámicas del espacio forestal en la región: una regulación estricta de las repoblaciones en zona urbana y de núcleos rurales, llegando a su prohibición; y el fomento de la gestión de la biomasa vegetal en las zonas limítrofes, con programas de actuación subsidiaria por parte de la administración ${ }^{16}$.

13 Decreto 64/1995, de 7 de marzo, por el que se establecen medidas de prevención de incendios forestales.

14 Ley 5/2003, de 22 de abril, de medidas de prevención de los incendios forestales en las urbanizaciones sin continuidad inmediata con la trama urbana.

15 Decreto 86/2006, de 2 de mayo, por el que se aprueba el Plan de Prevención de Incendios Forestales de la Comunidad Autónoma de Extremadura (Plan PREIFEX).

16 Decreto 105/2006, de 22 de junio, por el que se regulan medidas relativas a la prevención de incendios forestales, a la protección de los asentamientos en el medio rural y a la regulación de aprovechamientos y repoblaciones forestales. Ley 3/2007, de 9 de abril, de prevención y defensa contra los incendios forestales de Galicia. 
En definitiva, las escasas medidas preventivas desarrolladas por la normativa y los documentos de planificación autonómicos sobre la IUF se han planteado desde la doble consideración de la interfaz como un espacio vulnerable, en el que es necesario desarrollar actuaciones para proteger las personas y bienes de los incendios ${ }^{17}$, y generador de peligro, adoptando medidas dirigidas a evitar la ignición y posible propagación de incendios desde las zonas urbanizadas hacia las masas forestales.

\section{La incorporación de la variable de vulnerabilidad poblacional a los mapas de riesgo}

Como ya se ha señalado, sólo la delimitación de las zonas de riesgo por parte de la planificación sectorial (incendios, forestal) es vinculante para el resto de planificaciones. Por este motivo son particularmente relevantes los cambios que comienzan a operarse en algunas CCAA en la formación de estos mapas de riesgo, y ello en un doble sentido. Por un lado, desde el momento en que estos ejercicios de delimitación están evolucionando hacia la inclusión de nuevos parámetros de vulnerabilidad vinculados a la presencia de poblaciones y bienes potencialmente amenazados por incendios; y, por otro, por la manifiesta voluntad de declarar espacios que deben quedar al margen de los procesos de urbanización, mediante la correspondiente interpretación por la planificación urbanística de esta nueva cartografía de riesgo.

Las CCAA que más han avanzado en este sentido son las Islas Baleares, Cataluña y Galicia. La ley gallega de 2007 establece explícitamente que la zonificación del territorio según el riesgo espacial de incendio forestal debe incorporar el factor de la vulnerabilidad poblacional, al tiempo que señala la obligación por parte de los instrumentos de planeamiento urbanístico de incorporar las zonas de alto riesgo definidas por los planes de prevención y defensa contra los incendios forestales de distrito ${ }^{18}$. En esta evaluación se tiene en cuenta la densidad de población en zona forestal, el número de núcleos y la distancia entre ellos, así como la presencia de población de edad avanzada en ellos (Xunta de Galicia, 2009).

En este mismo sentido se desarrolla el Plan especial frente al riesgo de incendios forestales de las Islas Baleares (INFOBAL) ${ }^{19}$. En este caso, el cálculo de la vulnerabilidad poblacional incorpora los factores de ocupación (presencia de edificaciones en suelo forestal), colindancia (contacto de áreas urbanizadas con áreas forestales) y dispersión (proximidad o lejanía entre los diferentes asentamientos de población). Estos cálculos, además de informar sobre las prioridades de las medidas de prevención y de las dificultades para la extinción en estos ámbitos, tienen gran interés para el desarrollo de los planes de autoprotección, cuyas

17 En esta misma línea avanza el Código Técnico de la Edificación. En concreto, el Documento Básico SI de Seguridad en caso de incendio (2006) señala que en las zonas edificadas limítrofes o interiores a áreas forestales, deben cumplirse las condiciones siguientes:

a) Debe haber una franja de $25 \mathrm{~m}$ de anchura separando la zona edificada de la forestal, libre de arbustos o vegetación que pueda propagar un incendio del área forestal así como un camino perimetral de $5 \mathrm{~m}(\ldots)$;

b) La zona edificada o urbanizada debe disponer preferentemente de dos vías de acceso alternativas (...);

c) Cuando no se pueda disponer de las dos vías alternativas indicadas en el párrafo anterior, el acceso único debe finalizar en un fondo de saco de forma circular de $12,50 \mathrm{~m}$ de radio (...).

18 Ley $3 / 2007$, arts. 11 y 23.

19 Decreto 41/2005, de 22 de abril, por el que se aprueba el Plan especial frente al riesgo de incendios forestales. 
determinaciones deben ser incorporadas por el planeamiento urbanístico a través del planeamiento parcial para la ampliación o nueva creación de asentamientos urbanos sobre zonas o entornos forestales ${ }^{20}$. En términos similares se desarrolla la determinación de la vulnerabilidad en la normativa catalana, que igualmente establece parecidas obligaciones para el planeamiento en relación con los planes de autoprotección.

\section{La planificación de la emergencia}

Las especiales circunstancias en las que se desarrollan los fuegos de interfaz (riesgo para personas y bienes no forestales, evacuaciones, múltiples emergencias simultáneas) hacen de este tipo de incendios una preocupación creciente para los sistemas de extinción, que cada vez deben dedicar más tiempo y medios a la lucha frente a estas contingencias. Afrontar este nuevo escenario del fuego, en el que a las elevadas intensidades que puede alcanzar se une la afección a vidas humanas, debe superar además el problema añadido de la pérdida de toda una tradición de respeto hacia el peligro de incendio por arte de los residentes de estos espacios (muy especialmente en el caso de las urbanizaciones), lo que complica aún más la situación (Castellnou et al., 2005). En este contexto, las principales líneas de trabajo en la planificación de la emergencia pasan no sólo por el aumento de la eficacia de los dispositivos de extinción sino también, y muy especialmente, por la sensibilización de los habitantes en este tipo de espacios.

\section{Concienciación y autoprotección de los habitantes de la IUF}

El nivel de concienciación de la situación de riesgo que poseen los habitantes de la IUF en España es, en general, alarmantemente bajo. Esta falta de conciencia es especialmente notable en el caso de urbanizaciones exentas (en muchos casos de segunda residencia) situadas en zonas forestales, campings, y otros entornos asociados a la práctica vacacional, habitados por una población de características marcadamente urbanas; pero también afecta a núcleos rurales que hasta hace poco tiempo nunca habían sido afectados por los incendios forestales, pero que actualmente son vulnerables en relación con los cambios en la cubierta vegetal provocados por la falta de gestión del entorno más próximo (abandono de prácticas agrarias).

La necesidad de involucrar a la población residente es un factor clave para un proceso de gestión de riesgos satisfactorio. En este sentido, los esfuerzos de sensibilización persiguen un doble objetivo: proporcionar información que pueda conducir a un cambio en el comportamiento de las personas de manera que respondan a los riesgos de forma activa, y aportar los conocimientos necesarios para reaccionar adecuadamente en caso de peligro (Fleischauer et al., 2007). Sin embargo, son muy escasas las CCAA que han planteado campañas de este tipo (Cataluña, Canarias).

Además de la propia concienciación de vivir en un territorio de riesgo, estas campañas pretenden fomentar conductas de adaptación al riesgo por parte los propietarios tendentes a disminuir la vulnerabilidad de las estructuras. Estas prácticas se refieren a la propia edifica-

20 Decreto 41/2005, 1.7. 
ción (recomendaciones de emplazamiento, evitando las posiciones más peligrosas; uso de materiales resistentes al fuego; mantenimiento), al entorno inmediato (interrumpir la continuidad del combustible, reduciendo y controlando la vegetación de los jardines) y a la zona forestal exterior (rebajar la intensidad mediante podas, aclareos, limpieza del sotobosque).

El otro gran objetivo de la sensibilización consiste en informar sobre el comportamiento más adecuado durante los incendios. En este sentido es de gran importancia seguir las indicaciones de las autoridades sobre evacuaciones (procesos siempre delicados: la mayor parte de los fallecimientos se producen en huidas inadecuadas), o bien confinarse en la propia vivienda, para lo cual deben seguirse unas determinadas normas de comportamiento, durante y después del paso del fuego.

\section{El manejo de un nuevo escenario del fuego forestal}

Como ya se ha señalado anteriormente, el propio concepto de IUF designa, antes que nada, un escenario del fuego forestal que requiere de un tratamiento específico por parte de los sistemas de extinción que se aleja de la práctica habitual en los fuegos puramente forestales o urbanos (Davis, 1990). La necesidad de proteger simultáneamente personas y bienes, además de la propia masa forestal, obliga habitualmente a tomar decisiones comprometidas en el establecimiento de los objetivos prioritarios (Caballero, 2001), pues la obligación de atender preferentemente la protección de vidas humanas y bienes personales conduce a una pérdida de iniciativa en el ataque al fuego, lo que facilita la propagación del incendio por las masas forestales. Y todo ello, en un contexto de presión social máxima (Castellnou et al., 2005).

El análisis de incendios acaecidos en campañas con problemas graves de incendios urbano forestales (Cataluña, 2003) aconsejan cambios tácticos y de formación en los equipos de extinción para adaptarse a una nueva realidad caracterizada por la simultaneidad de emergencias (Castellnou et al., 2007). Especialmente delicadas son las decisiones sobre evacuación o confinamiento. En España no se puede hablar de estrategias diferentes previamente definidas (como sucede en Australia bajo el lema 'Prepare, stay and defend, or leave early', o en California) (Stephens et al., 2009), sino de protocolos de actuación que optan claramente por evacuar como solución preferente. Ello provoca no pocos enfrentamientos con la población, especialmente en zonas rurales, pues sus habitantes no aceptan con facilidad no poder defender directamente sus propiedades frente al fuego (Canarias, 2007).

\section{EL PROBLEMA DE LA IUF: ENTRE LA INSOSTENIBILIDAD DE LA DISPERSIÓN URBANA Y EL ABANDONO AGRÍCOLA}

Los avances en el conocimiento de esta realidad emergente en nuestro país no se han traducido hasta el momento en una percepción social extendida que considere la interfaz urbano-forestal como un territorio de riesgo. El incremento de episodios que afectan cada vez con mayor frecuencia a zonas habitadas (Canarias, 2007; Galicia, 2006; Cataluña, 2003) no se relaciona directamente con determinadas dinámicas territoriales que incrementan la vulnerabilidad del territorio. En concreto con el incremento de la urbanización dispersa en el medio rural, tanto en ámbitos metropolitanos como asociados al desarrollo turístico, y con 
los procesos de abandono agrícola en ámbitos especialmente sensibles (entornos de poblaciones).

La extendida opinión sobre la insostenibilidad de la creciente dispersión del modelo de asentamientos (planteada en términos de ineficiencia energética, segregación social, despilfarro territorial, fragmentación de hábitats, deterioro paisajístico, etc.) no se relaciona con su contribución al incremento de las situaciones de riesgo asociadas a los incendios forestales, y por el momento son muy limitadas las actuaciones dirigidas a disminuir su incidencia. Menos aún se es consciente de cómo los procesos de abandono y de cambios en las prácticas agrarias en los entornos de los núcleos habitados están modificando sustancialmente las pautas de propagación de los incendios forestales.

En conclusión, a pesar del interés y notoriedad que esta realidad ha ido progresivamente adquiriendo, todavía no se dan las condiciones necesarias y suficientes para una adecuada consideración de este nuevo territorio de riesgo:

- Ni de percepción social, lo cual inhibe en buena medida que se desarrolle un interés político por estas cuestiones,

- ni tampoco de capacidad técnica para caracterizar, delimitar y consiguientemente, evaluar y gestionar esta realidad con el desarrollo de instrumentos de planificación y ordenación eficaces.

\section{BIBLIOGRAFÍA}

AGER, A.A., VAILLANT, N.M. y FINNEY, M.A. (2010): «A comparison of landscape fuel treatment strategies to mitigate wildland fire risk in the urban interface and preserve old forest structure». Forest Ecology and Management, n ${ }^{\circ} 259$, págs. 1.556-1.570.

AVALAPATI, J.R.R., CARTER, D.R. y NEWMAN, D.H. (2005): «Wildland-urban interface: Challenges and Opportunities». Forest policy and Economics, ${ }^{\circ} 7$, págs. 705-708.

BADIA, A., SAURÍ, D., CERDÁN, R. y LLURDÉS, J.C.(2002): «Causality and management of forest fires in Mediterranean environments: an example from Catalonia». Environmental Hazards, $\mathrm{n}^{\circ} 4$ (2002), págs. 23-32.

BADIA, A., TULLA, A.F. y VERA, A. (2010): «Los incendios en zonas de interfase urbanoforestal. La integración de nuevos elementos en el diseño de la prevención». Scripta Nova. Revista electrónica de Geografía y Ciencias Sociales, Vol. XIV, no 331 (60).

BERINGER, J. (2000): «Community fire safety at the urban/rural interface: The bushfire risk». Fire Safety Journal, n ${ }^{\circ}$ 35, págs. 1-23.

BRANDIS GARCÍA, D. (2007): «Los espacios residenciales españoles en el cambio de siglo», en DELGADO VIÑAS, C. et al. (coords.): Espacios públicos, espacios privados: un debate sobre el territorio (XIX Congreso de Geógrafos Españoles, celebrado en Santander 26-29 de octubre de 2005), Universidad de Cantabria, AGE, Comunidad de Cantabria, págs. 25-53

BUTLER, C.P. (1976): «The urban/wildland fire interface». Fire Prevention Notes, California Department of Forestry, $\mathrm{n}^{\circ} 10$.

CABALLERO, D. (2001): «Particularidades del incendio forestal en el interfaz urbano. Caso de estudio en la Comunidad de Madrid». II Jornadas de Prevención de Incendios Forestales. ETSI Montes, Madrid. 
CABALLERO, D., BELTRÁN, I. y VELASCO, A. (2007): «Forest fires and WildlandUrban Interface in Spain: Types and Risk Distribution». Actas de la IV Conferencia Internacional de Incendios Forestales, Sevilla (España), 13-18 de mayo de 2007. Sesión Temática ${ }^{\circ} 5$.

CASTELLNOU, M., RODRÍGUEZ, L. y MIRALLES, M. (2005): «El problema de las urbanizaciones y el fuego forestal. Aportaciones desde la experiencia en Cataluña durante la campaña forestal del 2003». II Conferencia Internacional sobre Estrategias de Prevención de Incendios. Barcelona. 9-11 Mayo 2005.

CASTELlNOU, M., IGLESIAS, N., GUARQUE, J., PERNA, J. y LLEONART, S.(2007): «Experiencias de formación continuada en Cataluña durante las campañas 2003, 2004, 2005 y 2006». Actas de la IV Conferencia Internacional de Incendios Forestales, Sevilla (España), 13-18 de mayo de 2007. Sesión Temática $\mathrm{n}^{\circ} 7$.

DAVIS, J.B. (1990): «The wildland-urban interface: paradise or battleground?». Journal of Forestry, $\mathrm{n}^{\circ}$ 6, vol. 88 (1), págs. 26-31.

DUMAS, E., JAPPIOT, M. y TATONI, T. (2008): «Mediterranean urban-forest interface classification (MUFIC): A quantitative method combining SPOT5 imagery and landscape ecology indices». Landscape and Urban Planning, nº 84, págs. 183-190.

EUROPEAN COMMISSION (2006): Urban sprawl in Europe. The ignored challenge, Copenhagen, European Environment Agency, 56 págs.

EZQUIAGA, J.M. (2009): «Nuevo territorio y crisis del paradigma de la planificación convencional» en SÁNCHEZ PÉREZ-MONEO, L. y TROITIÑO VINUESA, M.A. (coords.): Agua, territorio y paisaje: de los instrumentos programados a la planificación aplicada: V Congreso Internacional de Ordenación del Territorio, págs. 563-578.

FLEISCHHAUER, M., GREIVING, S. y WACZURA, S. (2007): «Planificación territorial para la gestión de riesgos en Europa». Boletín de la Asociación de Geógrafos Españoles, $\mathrm{n}^{\circ} 45$, págs.. 49-78.

FONT ARELLANO, A. (2003): Planeamiento urbanístico. De la controversia a la renovación. Barcelona, Diputación.

FONT ARELLANO, A. (2007): La explosión de la ciudad. Transformaciones territoriales en las regiones urbanas de la Europa Meridional. (Catálogo de exposición) Barcelona: Ministerio de Vivienda: Collegi Oficial d'Arquitectes de Catalunya, 408 págs.

FOOTE, E., MARTIN, R. y GILLES, K. (1991): «The defensible space factor study: a survey instrument for post-fire structure loss analysis», en ANDREWS, P.L. \& POTTS, D.F. (eds.): Proceedings 11th Conference on Fire and Forest, págs. 66-73.

GALIANA MARTÍN, L., ARAGONESES DOMÍNGUEZ, C., MONTIEL MOLINA, C., DE LA CITA BENITO, F. y FERNÁNDEZ RAMIRO, M. (2009): «Caracterización de los escenarios del fuego en España», en $5^{\circ}$ Congreso Forestal Nacional. Montes y sociedad: saber qué hacer. S.E.C.F.-Junta de Castilla y León, Ávila, 21-15 de septiembre de 2009. Ref. 5CFE01-450.

GALIANA MARTÍN, L., HERRERO, G. y SOLANA, J. (2011): «A wildland-urban interface typology for forest fire risk management in Mediterranean areas». Landscape Research. Vol. 36, № 2, págs. 151-171.

GARCÍA LUCAS, A. y CARRASCAL TIRADO, J. (2007): «Planes Periurbanos de Prevención de Incendios Forestales en la interfaz urbano forestal en la Comunidad Autónoma de 
Extremadura». Actas de la IV Conferencia Internacional de Incendios Forestales, Sevilla (España), 13-18 de mayo de 2007. Sesión Temática ${ }^{\circ} 5$.

GONZÁLEZ, J.R. y PUKKALA, T. (2007): «Characterization of forest fires in Catalonia (north-east Spain)». European Journal of Forest Research, nº 126, págs. 421-429.

GREENPEACE (2009): El futuro en llamas. Cambio climático y evolución de los incendios forestales en España [Agosto 2009. Autores: Carlos Almagro y equipo de la campaña de Bosques y Clima]. www.greenpeace.es

HAMMER, R.B., RADELOFF, V.C., FRIED, J.S. y STEWART, S.I. (2007): «Wildlandurban interface housing during the 1990s in California, Oregon and Washington». International Journal of Wildland Fires, n ${ }^{\circ} 16$, págs. 255-265.

HAYNES, K., HANDMER, J., McANENEY, J., TIBBITS, A. y COATES, L.(2010): «Australian bushfire fatalities 1900-2008: exploring trends in relation to the 'Prepare, stay and defend or leave early' policy». Environmental Science \& Policy, nº 13, págs. 185-194.

INDOVINA, F. (2007): La ciudad de baja densidad: lógicas, gestión y contención, Barcelona, Diputació de Barcelona-Xarxa de municipis, 540 págs.

Informe sobre a investigación de incendios forestais en Galicia. Verán 2006 [Zona de Garda Civil de Galicia].

LAMPIN, C., JAPPIOT, M., LONG, M., MANSUY, N. y BORGNIET, L.(2006): «WUI and road networks/vegetation interfaces characterizing and mapping for forest fire risk assessment». Forest Ecology and Management, no 234 (Suppl. 1), págs. S137-S140.

LAMPIN-MAILLET, C., JAPPIOT, M., LONG, M., BOUILLON, Ch., MORGE, D. y FERRIER, J.-P.(2010): «Mapping wildland-urban interfaces at large scales integrating housing density and vegetation aggregation for fire prevention in the South of France». Journal of Environmental Management, ${ }^{\circ}$ 91, págs. 732-741.

MATA OLMO, R. (2007): Auge inmobiliario y evolución de los usos del suelo en España. Por una nueva cultura del territorio (Apertura del Curso Académico 2007-2008. Lección inaugural). Madrid, Universidad Autónoma de Madrid, 70 págs. http://www.uam.es/personal_pdi/filoyletras/rmata/

MMARM (2008): Los incendios forestales en España. Año 2007. Ministerio de Medio Ambiente y del Medio Rural y Marino.

MONTIEL, C. y HERRERO, G. (2010): «Overview of policies and practices related to fire ignitions», en SANDE, J. et al. (eds.): Towards Integrated Fire Management-Outcomes of the European Project Fire Paradox, European Forest Institute, págs. 35-46.

OBSERVATORIO DE LA SOSTENIBILIDAD EN ESPAÑA (2006): Cambios de ocupación del suelo en España: implicaciones para la sostenibilidad (estudio realizado a partir del proyecto Corine Land Cover). Madrid, Mundi-Prensa, 485 págs.

PINCETL, S., RUNDEL, P.W., CLARK, J., SILVER, D., SCOTT, T., KEELEY, J.E. y HALSEY, R.(2008): «It's the land use, Not the Fuels: Fires and Land Development in Southern California». Real Estate Review, Vol. 37 (1), págs. 25-42.

Proceedings of the International Scientific Workshop on «Forest Fires in the Wildland-Urban Interface and Rural Areas in Europe: an integral planning and management challenge» May 15 \& 16, 2003, Athens, Greece.

PYNE, S. J., ANDREW, P.L.y LAVEN, R.D. (1996): «Introduction to Wildland fire». New York, Wiley, 769 págs. 
RADELOFF, V. C., HAMMER, R.B., STEWART, S.I., FRIED, J.S., HOLCOMB, S.S. y McKEEFRY, J.F.(2005). «The wildland-urban interface in the United States». Ecological Applications, $\mathrm{n}^{\circ} 15$ (3), págs. 799-805.

REAMS, M.A., HAINES, T.K., RENNER, C.R., WASCOM, M.W. y KINGRE, H.(2005): «Goals, obstacles and effective strategies of wildfire mitigation programs in the Wildland-Urban Interface». Forest Policy \& Economics, n ${ }^{\circ}$, págs. 818-826.

REINHARDT, E.D., KEANE, R.E., CALKIN, D.E. y COHEN, J.D.(2008): «Objectives and considerations for wildland fuel treatment in forested ecosystems of the interior western United States». Forest Ecology and Management, ${ }^{\circ}{ }^{\circ}$ 256, págs. 1.997-2.006.

RIFÀ, A. y CASTELLNOU, M. (2007): «El modelo de extinción de incendios forestales catalán». Actas de la IV Conferencia Internacional de Incendios Forestales, Sevilla (España), 13-18 de mayo de 2007. Sesión Temática $n^{\circ} 2$.

SAFFORD, H.D., SCHMIDT, D.A. y CARLSON, C.H. (2009): «Effects of fuel treatments on fire severity in an area of wildland-urban interface, Angora Fire, Lake Tahoe Basin, California». Forest Ecology and Management, n 258, págs. 773-787.

STEPHENS, S.L., ADAMS, M.A., HANDMER, J., KEARNS, F.R., LEICESTER, B., LEONARD, J. y MORITZ, M.A. (2009): «Urban-wildland fires: how California and other regions of the US can learn from Australia». Environmental Research Letters, ${ }^{\circ}$ 4, 5 págs.

TERÉS BLANCO, J.A., LLAQUET NADAL, J., TUDELA PIÑOL, A., CANYAMERES RAMONEDA, E. (2007): «El tratamiento de la vegetación para la prevención de incendios forestales en la interfaz urbano-forestal en Catalunya». Actas de la IV Conferencia Internacional de Incendios Forestales, Sevilla (España), 13-18 de mayo de 2007. Sesión Temática $\mathrm{n}^{\mathrm{o}} 5$.

USDA \& USDI (2001). »Urban wildland interface communities within vicinity of Federal lands that are at high risk from wildfire». Federal Register, n 66, págs. 751-777.

VILAR DEL HOYO, L., MARTÍN ISABEL, M.P. y MARTÍNEZ VEGA, J. (2008): «Empleo de técnicas de regresión logística para la obtención de modelos de riesgo humano de incendio forestal a escala regional». Boletín de la Asociación de Geógrafos Españoles, $\mathrm{n}^{\circ} 47$, págs. $5-29$.

VINCE, S.W., DURYEA, M.L., MACIE, E.A. y HERMANSEN, A. (eds.) (2005): Forests at the wildland-urban interface: conservation and management. Boca Raton, CRC Press, 293 págs.

WALT, H.R. (1988): «Current and Future Wildland Fire Protection Impacts of the WildlandUrban Interface», en BERG, N.H. (Coord.): Proceedings of the Symposium on Fire \& Watershed Management (October, 26-28, 1988. Sacramento, California), págs. 3-7.

XUNTA DE GALICIA (2006): Informe sobre a vaga de incendios forestais do mes de agosto de 2006. [Xunta de Galicia. Consellería do Medio Rural. Dirección Xeral de Montes de Industrias Forestais]. 30 de agosto de 2006.

XUNTA DE GALICIA (2009): PLADIGA 2009 (Plan de Prevención e Defensa contra os Incendios Forestais de Galicia). [Xunta de Galicia. Consellería do Medio Rural. Dirección Xeral de Montes].

ZHANG, Y., HE, H.S. y YANG, J.(2008): «The wildland-urban interface dynamics in the southeastern U.S. from 1990 to 2000». Landscape and Urban Planning, $\mathrm{n}^{\circ}$ 85(3), págs. 155-162. 